\title{
When Adolescents Feel Ugly: Cognitive Behavioral Therapy for Body Dysmorphic Disorder in Youth
}

\author{
Cynthia Turner, PhD \\ School of Psychology, Australian Catholic University, Brisbane, Australia \\ Jacinda Cadman, BA (Hons), MS \\ Griffith University, Gold Coast, Australia
}

\begin{abstract}
Adolescence is a critical time for physical development and maturation, and with these important physiological changes comes greater awareness of body image and appearance, which, for a proportion of young people can become excessive, signaling the onset of body dysmorphic disorder (BDD). BDD in adolescence is associated with significant impairment and suicidality, is poorly understood, and currently there is limited evidence for the effectiveness of psychological therapy. Cognitive behavioral therapy (CBT) is currently the most promising and best available psychological therapy for BDD in youth. The aim of this article is to provide clinicians with information on CBT treatment for BDD in young people and to provide guidance based on clinical experience of working with this complex population group. The article will include discussion of strategies including maximizing psychoeducation with parental involvement, the use of cognitive therapy techniques, exposure with response prevention, and perceptual visual training techniques, including attention training and mirror retraining.
\end{abstract}

Keywords: body dysmorphic disorder; adolescence; youth; cognitive behavioral therapy

\begin{abstract}
dolescence is a stage of rapid growth and development, with distinctive changes in physical appearance and cognitive development. Concern about body image and appearance becomes apparent, and appearance evaluation tends to increase (Littleton \& Ollendick, 2003; Mitchell, Petrie, Greenleaf, \& Martin, 2012). Society too has become increasingly preoccupied with physical appearance and beauty, with this fixation apparent across a myriad of print and online media, pervasively advocating to youth the importance of appearance and body image (Cash \& Pruzinsky, 2002). Dissatisfaction with body image seems so widespread in Western society that it could be argued to be normative, and Rodin, Silberstein, and StreigelMoore (1984) coined the phrase "normative discontent" to describe pervasive feelings of body image dissatisfaction. Body dissatisfaction becomes apparent from 12 to 15 years of age for many adolescents (Levine \& Smolak, 2002). For some, however, appearance concerns are intensified to such an extent that their capacity to function in everyday life is compromised, and evidence suggests the typical age of onset for body dysmorphic disorder (BDD) occurs during adolescence (e.g., Gunstad \& Phillips, 2003; Phillips, Didie, et al., 2006).
\end{abstract}


Although appearance concerns are a typical and inherent part of adolescence, adolescents with BDD differ from their peers in terms of both the significant distress caused by the preoccupation with their appearance and the time spent engaging in the repetitive behaviors that relate to these preoccupations (Greenberg et al., 2010; Lambrou, Veale, $\&$ Wilson, 2012). Adolescents with BDD will commonly spend between 3 and 8 hours daily engaged in repetitive behaviors and/or attempting to camouflage their perceived defect. Adolescents with BDD tend to experience high rates of suicidal ideation, with one study finding that $44.4 \%$ of adolescents had attempted suicide (Phillips, Didie, et al., 2006), and this appears to be more frequent than in adults with the disorder (Phillips \& Diaz, 1997). Adolescents tend to display a higher degree of delusion toward their focal areas of preoccupation and display less insight into their thoughts and behavior (Phillips, Didie, et al., 2006). The prevalence of BDD symptoms in adolescents is $1.7 \%-2.3 \%$, with few significant gender differences (Schneider, Mond, Turner, \& Hudson, 2017a; Schneider, Turner, Mond, \& Hudson, 2017), although prevalence rates tend to be higher for older adolescents as compared with younger adolescents (Rief, Buhlmann, Wilhelm, Borkenhagen, \& Brähler, 2006; Schneider et al., 2017).

Subclinical or subthreshold BDD symptoms are more prevalent again, with 3.4\% of adolescents reporting dysmorphic concerns that cause distress or impairment (Schneider, Mond, Turner, \& Hudson, 2017b). These subclinical BDD symptoms are associated with high levels of anxiety and depression, and reduced quality of life, suggesting that even milder forms of BDD preoccupation do not represent a normal level of appearance concern (Schneider et al., 2017b). What remains unknown at present is the course of milder BDD symptoms over time. It may be that many of these young people do not progress on to develop more intense symptoms over time, or conversely, they may do. These sobering findings underscore the importance of better understanding this debilitating disorder.

\section{Cognitive-Behavioral Model}

To effectively understand and ultimately treat BDD, researchers have begun to delineate the underlying mechanisms and processes that may contribute to the development and maintenance of the disorder (e.g., Neziroglu, Roberts, \& Yaryuya-Tobias, 2004; Veale, 2004). This theoretical conceptualization and understanding of BDD is most notably documented in the cognitive-behavioral model of the disorder proposed by researchers Veale, Wilhelm, and colleagues (e.g., Neziroglu, Khemlani-Patel, \& Veale, 2008; Veale, 2004; Wilhelm, Buhlmann, Hayward, Greenberg, \& Dimaite, 2010). The model seeks to understand the maintaining processes relevant to $\mathrm{BDD}$, which ultimately assists in guiding the development of treatment strategies. At the core of the model lies the notion of processing oneself as an aesthetic object (Veale, 2004), or having physical appeal. For those with BDD, viewing oneself in this way involves an experience of engaging in extreme self-focused attention onto one's own (distorted) body image. Veale and colleagues purport that this process of mental imagery is triggered by a particular event that activates a representation of the person's appearance. The event can be either external (e.g., seeing an image of oneself in a mirror, a comment from another person) or internal (e.g., an intrusive thought about their negative appearance). The process of selective attention facilitates this experience, having the effect of increasing awareness of, and engagement with, the (distorted) mental image and of particular features within that image. In turn, this activates a negative body image appraisal that focuses on assumptions regarding the importance of one's appearance.

For those with BDD, there is a tendency to associate physical appearance with their core identity and worth (Veale, 2002a). Such assumptions are based on core beliefs regarding inadequacy, worthlessness, repulsiveness, loneliness, and rejection. These assumptions may manifest 
themselves as declarations such as "If I am unattractive, then I will be alone all my life," "How I feel about myself as a person is related to how I feel about how I look," or "If I look better, my whole life will be better" (Geremia \& Neziroglu, 2001; Veale et al., 1996). From this negative selfappraisal stems various maladaptive cognitive and behavioral responses, such as safety-seeking behaviors to camouflage and/or alter the appearance flaw; mood changes, particularly regarding shame, disgust, depression, and anger; and rumination on the defective image spurred by intrusive thoughts (Veale, 2002b).

There is considerable research effort required to determine the empirical validity of a disorder-specific model, and evidence in support of the cognitive-behavioral model of BDD is accumulating. For example, Osman, Cooper, Hackmann, and Veale (2004) examined the experience of mental imagery in individuals with BDD and found that mental self-images were rated as significantly more negative, recurrent, and vivid in individuals with BDD as compared to a control group. Evidence suggests that individuals with BDD are more likely to view themselves aesthetically and to value physical appearance more highly than art and design students and healthy controls (Lambrou, Veale, \& Wilson, 2011). Buhlmann, Etcoff, and Wilhelm (2006) examined the recognition and interpretation of emotional expressions, finding that participants with $\mathrm{BDD}$, relative to controls, misinterpreted emotional expressions as angry and contemptuous in "self-referent" but not in "other-referent" situations, suggesting a negative interpretation bias for others emotional expressions. Studies have further examined the role of appearance-related rumination and selective attention, finding more negative beliefs about appearance in participants with BDD as compared to controls (Buhlmann et al., 2006), and greater attention to threatening stimuli (Buhlman, McNally, Wilhelm, \& Florin, 2002).

\section{Cognitive Behavioral Therapy for BDD in Adults}

The cognitive-behavioral model has significant clinical utility because it outlines important psychological factors that are responsible for maintenance of the disorder (e.g., repetitive behaviors, avoidance, selective attention) and then seeks to target these via the development of treatment strategies that will reverse these maintaining factors. Most cognitive behavioral therapy (CBT) studies employ exposure with response prevention (E/RP) strategies. E/RP typically involves encouraging an individual to refrain from engaging in repetitive behaviors or avoidance strategies, usually while being observed by others, and then using objective information to determine whether their BDD worries are supported. Other techniques include perceptual visual training techniques, such as mirror retraining. Perceptual mirror retraining is a visual training strategy that involves an individual viewing their entire face and body in a full-length mirror, while scanning down from head to toes, and describing each body part nonjudgmentally and objectively, and refraining from rituals or repetitive behavior (Wilhelm, Phillips, Fama, Greenberg, \& Steketee, 2011). Mirror training has also been used as a mirror exposure technique (e.g., Neziroglu, McKay, Todaro, Yaryura-Tobias, 1996). Wilhelm and colleagues (Wilhelm et al., 2011; Wilhelm, Phillips, \& Steketee, 2013) describe a modular approach to CBT treatment, which uses the core CBT elements of education, E/RP, perceptual retraining, and identifying and modifying appearance-related cognitions and beliefs, with additional optional modules to address client-specific symptoms (e.g., cosmetic surgery seeking, mood management).

There is accumulating evidence that CBT is an effective intervention for adults with BDD. To date, there have been six randomized controlled trials conducted examining the efficacy of CBT alone (Enander et al., 2016; Rabiei, Mulkens, Kalantari, Molavi, \& Bahrami, 2012; Rosen, Reiter, \& Orosan, 1995; Wilhelm et al., 2014; Veale et al., 2014; Veale et al., 1996), summarized in two recent meta-analytic reviews (Harrison, de la Cruz, Enander, Radua, \& Mataix-Cols, 2016; 
Phillipou, Rossell, Wilding, \& Castle, 2016). These meta-analyses indicate that CBT is superior to waitlist and credible psychological placebo in reducing BDD and depression symptoms, that CBT is associated with significant improvements in insight/delusionality, and that treatment gains are maintained over small follow-up periods of $2-4$ months.

\section{Cognitive Behavioral Therapy for BDD in Adolescents}

As is often the case, the literature pertaining to adolescent BDD lags significantly behind that pertaining to adults with the disorder. There are few experimental studies which seek to test etiological or maintaining factors in young people, and the current CBT treatment literature for $\mathrm{BDD}$ in adolescents remains limited at the time of writing. There are a small number of single case reports (e.g., Aldea, Storch, Geffken, \& Murphy, 2009; Greenberg et al., 2010) and two larger case series, incorporating 6 and 13 young patients, respectively (Greenberg, Mothi, \& Wilhelm, 2016; Krebs, Turner, Heyman, \& Mataix-Cols, 2012). These case studies/case series essentially describe the clinical presentation of BDD in young people and present preliminary, uncontrolled evidence that CBT is feasible and acceptable in this population group. Krebs et al. (2012) report on CBT treatment emphasizing E/RP and behavioral experiments for this population group, and Greenberg et al. (2016) report on a modular CBT protocol, not dissimilar to that developed for adults (Wilhelm et al., 2013), although incorporating significantly greater levels of family involvement and an adolescent-specific module focused on school refusal. These studies provide a basis for hypothesizing that developmentally tailored CBT can be effective and that treatment gains can be maintained through to 6-month follow-ups (e.g., Aldea et al., 2009; Greenberg et al., 2010; Krebs et al., 2012).

To-date, only one pilot randomized clinical trial has been conducted and reported for adolescents with BDD (Krebs et al., 2017; Mataix-Cols et al., 2015). Here, we will summarize the core components of this study, reporting the results and follow-up, and then discuss in more detail the treatment components relevant to cognitive behavioral therapy for youth with $\mathrm{BDD}$, drawing on case examples and vignettes from our clinical experience with this population group.

In developing the CBT protocol for the Mataix-Cols et al. (2015) study, we drew on our experience of treating young people with obsessive-compulsive disorder (OCD; e.g., Nakatani, Mataix-Cols, Micali, Turner, \& Heyman, 2009; Turner et al., 2014), and BDD (Krebs et al., 2012), in addition to other published material regarding adolescent BDD (e.g., Greenberg et al., 2010), and existing treatment protocols and trials for adult BDD (e.g., Veale et al. 2014; Veale \& Neziroglu, 2010). To support uptake by adolescents and their parents, we developed age-appropriate worksheets and handouts using simplified language and concepts. We sought to incorporate high levels of parental involvement, particularly when parents were highly accommodating of the disorder symptoms or when parental support of the young person was clinically required (e.g., if parents were critical of their child or if the young person felt they could not proceed with an assigned task without higher levels of involvement from a significant other). The initial 14-session treatment protocol consisted of three main phases: psychoeducation, E/RP (and/or behavioral experiments), and relapse prevention. We incorporated other treatment elements as clinically required, primarily mirror retraining and attention training. All treatment tasks were initially completed in-session with therapist assistance and assigned for home practice between sessions. Relapse prevention strategies incorporated significant discussion of maintaining gains made and plans for continuing to improve. Given the pilot nature of the study, accommodations were made when young people required additional sessions, or more time to complete sessions.

The CBT treatment was compared to a control condition that provided participants with written materials containing age-appropriate information about BDD but did not include 
information regarding the psychological treatment of BDD. Participants were advised that they could e-mail or telephone the clinic with any questions, or to seek general support if required, and once a week, each participating family in the control condition were telephoned to monitor mood, risk, and suicidal ideation.

In total, 30 adolescents ranging in age from 12 to 18 years were randomly assigned to either the CBT or the control condition, and diagnostic and/or self-report assessments were undertaken at pretreatment, midtreatment, posttreatment, and at 2- and 12-month follow-up (12-month followup separately reported; Krebs et al., 2017). Young people in the CBT condition showed significantly greater improvement at posttreatment and follow-up, with more than $50 \%$ of youth in the CBT condition rated as improved or much improved via blind clinician ratings. Symptom improvement was also observed on secondary measures of illness insight, depression, and quality of life. Following completion of the 2-month follow-up, participants allocated to the control condition were offered CBT treatment, and therefore, the 12-month follow-up is a naturalistic design and includes all participants receiving CBT $(N=26)$. At the 12 -month point, 13 out of 26 participants $(50 \%)$ were classified as treatment responders, and 6 participants (23.1\%) were classified as achieving disorder remission. Although these outcomes are promising, it also indicates that there remains significant scope to improve on treatment with improved knowledge and understanding of the disorder. Nonetheless, young people and their parents reported satisfaction with the treatment and found it acceptable. We will turn now to some of the insights gained from working with young people and to consider in more depth the components of CBT treatment with adolescents.

\section{CBT STRATEgIES FOR BDD Treatment For YOUTH}

As noted earlier, BDD treatment protocols for young people have been developed based on experience in working with OCD as well as BDD in youth and adapted from adult models of BDD and adult BDD studies. Modifications to adult treatment techniques have involved incorporating developmentally appropriate information, seeking to simplify some of the techniques, and to incorporate greater levels of parental/familial involvement to recognize the important role that family plays in a young person's life. These modifications apply to all three primary treatment phases: psychoeducation, E/RP, and relapse prevention. In the following text, we highlight some of the key learnings we have gleaned from our experiences in working with young people with BDD.

\section{Psychoeducation}

One of the main aims of psychoeducation in treatment with youth and their families is to develop a shared understanding of BDD. Key components of BDD psychoeducation for young people include the following: distinguishing between $\mathrm{BDD}$ and normal appearance and body image concerns, introducing and discussing the role of perception regarding body image, and outlining the cycle of BDD, emphasizing the role of safety behaviors, rituals and avoidance in maintaining ongoing distress, and thus providing a rationale for exposure-based therapy. It is important for young people to understand that exposure provides inhibitory learning opportunities and habituation through removal of safety behaviors and rituals. These complex processes are explained as simply as possible by drawing parallels with normative developmental experience of anxiety-provoking events or situations and subsequently emphasizing the way that BDD can accelerate or intensify these normative experiences.

Compared to working with adults, completing the psychoeducation phase of treatment with young people and families may require additional time. This can be because of various factors, such as level of understanding and insight of the young person and the extent of familial involvement and parental understanding. On occasion, if parents are critical of the behaviors that their child engages in, it can be difficult for them to acknowledge and accept that these are not volitional 
on the part of the adolescent. For the young person, understanding and accepting the psychoeducational phase of treatment can be negatively affected by poor insight and treatment motivation because for many young people, treatment may initially feel to them to be involuntary and at the insistence of their parents. Acknowledging this and using motivational interviewing techniques can be helpful and important in this initial phase. Overall, detailed and age-appropriate psychoeducation is important in providing a clear and solid framework for the treatment that follows.

\section{Parental Involvement}

Most of young people seeking treatment for BDD reside at home and require strong and ongoing family support to successfully complete their treatment program. Throughout treatment, but particularly during the psychoeducation phase, parental involvement is critical to ensure that families have a shared understanding of the young person's difficulties. During the psychoeducation phase, this is achieved through inclusive discussions regarding the young person's idiosyncratic BDD formulation. An idiosyncratic formulation will include probable causes of BDD, the evolution of anxiety, and the cycle of BDD. It is important, when developing a personalized cycle of maintaining factors for BDD, that distorted beliefs about appearance as well as rituals and avoidance strategies that are aimed at reducing, fixing, or camouflaging appearance flaws and neutralizing distress are included. An example of a simple BDD cycle for an adolescent is depicted in Figure 1.

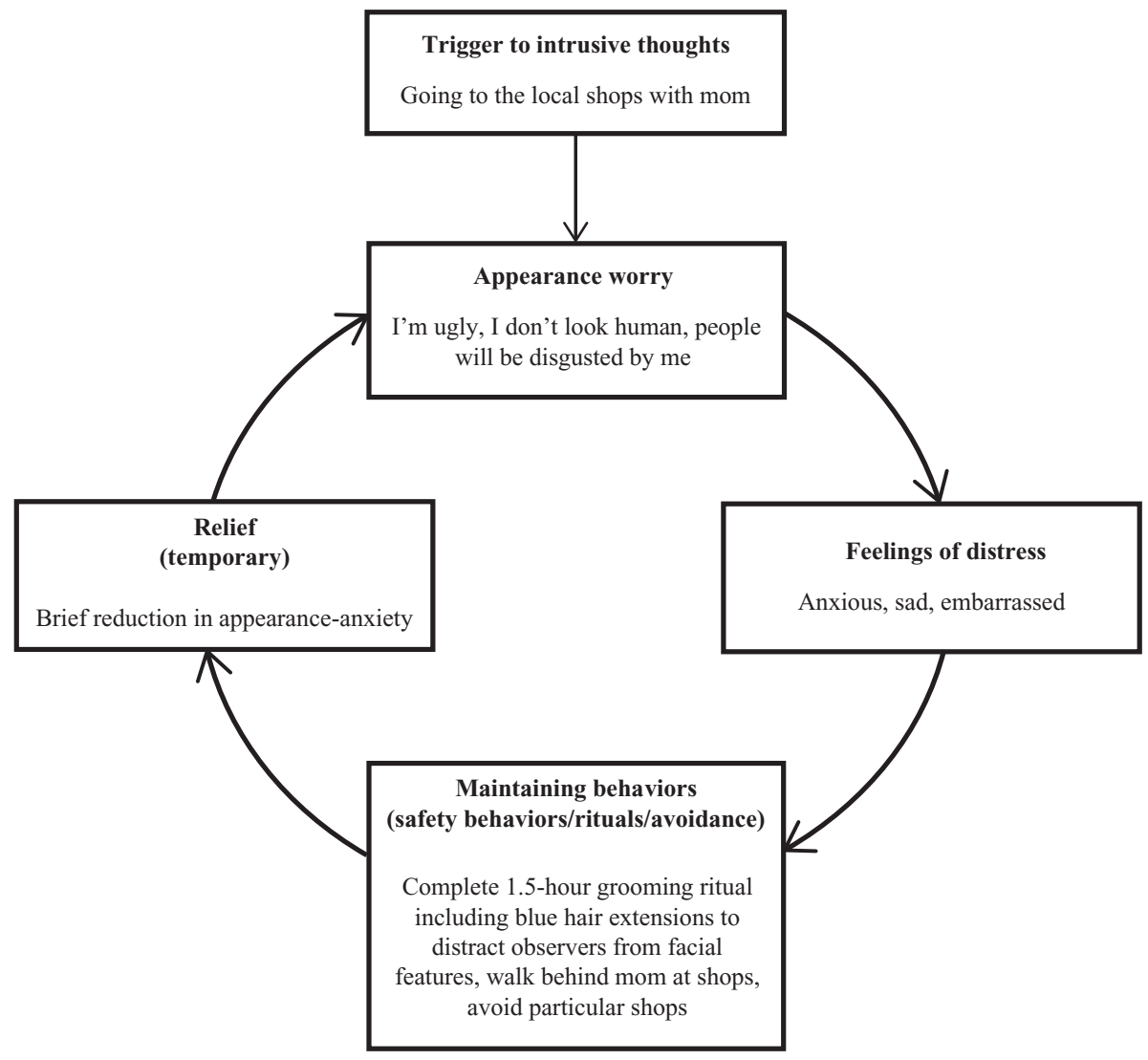

FIGURE 1. An example of an idiosyncratic body dysmorphic disorder cycle for an adolescent with appearance intrusions regarding facial features. 
Family accommodation of a young person's symptoms is a widely recognized phenomenon in families of OCD sufferers (e.g., Lebowitz, Panza, Su, \& Bloch, 2012). Family accommodation of a young person's BDD symptoms appears to be similarly common. Examples of accommodation include parents purchasing makeup for their child, parents scheduling specialist appointments (e.g., with cosmetic surgeons or dermatologists), providing repeated reassurance about appearance worries, paying for costly appearance-related items (e.g., colored contact lenses, hair extensions, false eye lashes, particular clothes), and enabling or facilitating avoidance of social situations, such as facilitating avoidance of sports classes at school by writing notes to school personnel. Educating the family about the rationale for treatment, emphasizing that this will include a gradual reduction in accommodation of rituals and avoidance, helps parents to feel more confident in supporting their child through treatment while reassuring the young person that treatment will progress at a manageable pace.

\section{Determining Whether Appearance Concerns Are Normal or Excessive}

Typical adolescent development sees a notable intensification in awareness of body image and appearance, increasingly reinforced by various media and popular culture. It is fundamentally important, therefore, that treatment discussions should seek to distinguish between normal appearance concerns in young people and intrusive, distressing appearance-related intrusions and worries. Drawing on a young person's knowledge of his or her peers' grooming behaviors, and/or surveying similarly aged peers with personally relevant questions, can be used as a basis for determining typical and atypical levels of grooming behavior (Table 1). This information can then be used to develop specific and realistic goals for treatment. More important, distinguishing between normal and excessive adolescent appearance worries reinforces for the young person that their concerns are not as a result of vanity but are attributable to a known and understood psychological disorder. This knowledge can help to reduce a young person's feelings of shame and isolation, reduce feelings of embarrassment about disclosing their difficulties, and increase the feeling of being understood.

\section{Exposure With Response Prevention}

Although the small number of adolescent BDD treatment studies do not allow for examination of differential treatment effects, our inclination at this early stage of treatment development is that graduated exposure to feared situations has the most significant impact on BDD symptom reduction in young people. The degree of graduation between tasks tends to be smaller than what we may seek in treating youth with OCD, particularly in the earliest stages of E/RP. Subjective distress in youth with BDD tends to be very high, and comorbid depression with suicidal ideation is common. During E/RP, it is important for the therapist to be working actively to contain distress while facilitating opportunities for new learning to occur. If a young person worries that they will

TABLE 1. An Example of Typical Versus Atypical Grooming Behaviors in Adolescence

\begin{tabular}{|c|c|c|}
\hline Psychological Impact & $\begin{array}{l}\text { Typical Adolescent } \\
\text { Grooming Behavior }\end{array}$ & $\begin{array}{l}\text { Atypical Adolescent } \\
\text { Grooming Behavior }\end{array}$ \\
\hline Time spent & 45 min-1 hour & $>3$ hours \\
\hline Subjective distress rating & 3 out of possible 10 & 9 out of possible 10 \\
\hline Interference & None; 5 min late for engagement & Won't leave the house \\
\hline
\end{tabular}


TABLE 2. Some Examples of SMall Initial Exposure Tasks in Body Dysmorphic Disorder (BDD) TREATMENT FOR YOUTH

\begin{tabular}{ll}
\hline Ritual & Possible First Exposure Task \\
\hline Wearing hair over face & Tuck a few strands of hair behind ear. \\
Wearing baggy clothing & Wear slightly tighter shirt in bedroom. \\
Grooming routine lasting 2.5 hours & Reduce grooming routine to 2 hours and 20 min. \\
Sitting on chair with heels raised & Sit with flat feet for short period at home. \\
Mother to carry back-up makeup kit & Mother to remove one highlighter from kit \\
Situating self behind others in photographs & Standing behind shorter people in photo. \\
Comparing oneself to others on social media & Unfollow one person on Instagram. \\
\hline
\end{tabular}

be asked to engage in an exposure task that is beyond their perceived ability, their distress will become quickly and highly elevated and there is a risk that they will disengage from treatment. Encouraging judicious use of safety behaviors to facilitate completion of a small exposure task is often necessary during the initial stages of exposure therapy. Table 2 provides some examples of the very small graduations that may be appropriate for initial E/RP tasks.

Through behavioral experiments and E/RP tasks, accompanied with predictions about anticipated outcomes, young people are able to test out their appearance-related concerns, and gather successive pieces of evidence that will support an alternative conceptualization of their problem. That is, that they have a worry problem about appearance, rather than having an appearance problem. The example in the following text provides an outline of how an exposure task may be set up for a young person with concerns regarding the appearance of their upper arms.

Jennie had intrusive, distressing thoughts regarding the appearance of her upper arms. She feared that her arms were disgusting: too fleshy and pale. She held a belief that others would think she looked disgusting, that others would stare at her arms, and think she was a freak. She ensured that she always wore $t$-shirts that did not reveal any of her upper arms. In discussion with her therapist, Jennie agreed to test this by rolling up her shirt sleeves with one roll only, and walk through the psychology clinic where she was receiving therapy. She predicted that this would result in a moderate level of distress (5 out of 10) and that others would stare excessively at her arms, not glancing at her face at all. After completing this task with her therapist, Jennie concluded that no one had started at her arms, and that perhaps they didn't think her arms looked "freaky." Her distress peaked at 3.5 out of 10, and reduced within 10 minutes of completing the task. Jennie learned that she could cope with a small area of her arm on display.

Therapy with Jennie might involve repeating this task in various different settings (private settings initially and then increasingly more public) to generalize her learning and, as soon as she felt able, to increase the degree of exposure of her upper arm. When a young person's predictions about what may happen in a task are violated, his or her insight and belief in the treatment improves. In this way, more difficult exposure tasks completed later in treatment are deemed more acceptable because of early evidence gained.

It can be helpful to incorporate a reward schedule (i.e., contingency management) into therapy to provide some additional motivation for adolescents to engage in therapeutic task and homework tasks. Positive contingencies may be applied by the therapist for engagement in, and completion of in vivo E/RP tasks within sessions, and may also be applied for tasks completed between sessions. Contingency management is based on operant principles of behavior management, and to be effective, must be salient for the individual for whom they are being used. 


\section{The Inclusion of Cognitive Strategies}

In working with young people with BDD, we tend to emphasize behavioral strategies to a greater extent than when working with adults with the disorder. This preference is somewhat similar to the way in which we might approach OCD treatment with young people (e.g., Turner et al., 2014). Although cognitively based treatment strategies, such as cognitive restructuring and distancing thoughts, are still used alongside exposure tasks, one particular cognitive skill, namely imagery rescripting, is rarely used, despite its apparent efficacy in adult BDD treatment protocols (e.g., Willson, Veale, \& Freeston, 2016). In most instances, it is likely to be inappropriate and unhelpful to focus the young person on a salient memory. There are several reasons for this. Many young people are not able to gain an updated adult perspective on a social experience they experienced as traumatic. They may continue to be actively engaged in the social situation that they find distressing (e.g., school), they may be victimized within this setting in an ongoing way (e.g., being teased or bullied), they may be aware of others within their social environment having witnessed or participated peripherally in the traumatic event. Instead of imagery rescripting, empathizing with the young person's experience, and problem solving where appropriate, is recommended. Additional cognitive strategies that may be used in combination with exposure therapy include attention training, mirror retraining, and the use of surveys.

\section{Attention Training}

Attention training involves teaching young persons to redirect their attention away from internal worries and the (distorted) mind's eye image of themselves and on to external stimuli, using as many of their senses as possible. Instructing young persons to notice the things they can see, smell, feel, taste, and hear encourages them to shift their focus of attention away from their appearance and into the present moment. Many youth with BDD will hold beliefs that others judge them as disgusting and unattractive, and they move through social situations assuming this to be the case. By increasing young persons' volitional attentional control and flexibility, they are better able to notice cues in their social environment, which allows them to accumulate evidence that other people are not in fact staring, sneering, or grimacing as they pass by, thus disconfirming their BDD beliefs.

\section{Mirror Retraining}

The use of mirror retraining with adults has proved to be particularly helpful (Wilhelm et al., 2013) and translates well for use with young people with repetitive behaviors that involve checking aspects of their appearance in the mirror. Not only does mirror retraining reinforce appropriate mirror use or mirror hygiene (i.e., remaining a "safe" distance from the mirror for a short, defined time), but it also encourages young persons to view their image nonjudgmentally and as a whole, rather than focusing solely and harshly on their features of concern. This strategy also works as an exposure task for youth who avoid mirrors. By first focusing their gaze on a nonthreatening part of their body, young person are encouraged to practice describing their appearance in factual terms, before then being encouraged to shift their focus to another part of their body. Initially, adolescents are likely to struggle to use neutral, factual terms to describe their appearance, and therapists will need to role model this behavior. To make a start with a strategy such as this, therapists may elect to use a photograph of the adolescent, taken at a distance with a zoom lens, or to stand at some distance from the front of a full-length mirror. Eventually, after repeated practice and observation of a role model, the young persons should be able to describe previously distressing aspects of their appearance in a neutral tone and to shift to view their appearance as a whole, rather than remaining fixated on features of concern. For example, 
youth who previously spent hours throughout the day checking their appearance in a magnifying mirror to self-criticize their "huge, beaked" nose would be encouraged to remain more than $1 \mathrm{~m}$ from a full-length mirror and to describe their entire face as " ... round with a soft v-shape at the bottom, light brown skin, pinkish oval-shaped lips with a small dip on top, a nose that is a triangular shape approximately $2 \mathrm{~cm}$ deep, $3 \mathrm{~cm}$ wide, and $5 \mathrm{~cm}$ long, green colored eyes with black, brown and grey flecks ... and so on." Mirror retraining is a task that can be set as homework, with or without parental support depending on the individual child, to increase a young person's competence with this strategy.

\section{Surveys}

The use of surveys to gather evidence against BDD beliefs is useful when working with adolescents. Working with the young person to devise helpful questions that will target individualized dysmorphic concerns is important in order for surveys to be effective. This may include the therapist encouraging the adolescent to consider questions related to exploring values because simply gathering evidence that "others think I'm attractive" usually holds little to no weight for young people and is likely to be discounted for various reasons. Asking others to complete survey questions regarding what they value in other people, and how much of a role appearance plays in developing relationships, can be useful points of discussion and learning. For example, if an adolescent believes that looking unattractive means they will never make friends, the survey could ask whether physical appearance impacts on their friendship choices and what else they look for in a friend. The use of photos of the young person with BDD, to anchor specific questions about appearance, can also be helpful. For example, a survey accompanying a photograph to investigate whether other people notice a perceived flaw, may ask other people to comment on when and/or whether they also notice other features (i.e., "What do you first notice in this photograph?"; "Rate how bright the red in his cheeks appears to be using the following scale"). Therapists should make sure to confirm that survey responses are useful to therapy before presenting to the young person.

\section{Relapse Prevention}

Although young people are rarely symptom free during the final stages of psychological treatment, devising an ongoing plan for maintaining gains and preventing relapse is recommended. Our understanding of what may best predict recovery (and relapse) in youth with BDD is limited; however, it is considered that a relapse prevention plan should include ongoing E/RP tasks for any remaining rituals or repetitive behaviors to reach treatment goals. Symptoms that are likely to reemerge, and future situations where the young person may be vulnerable to a reemergence of symptoms, should be identified and steps to address these put in place, and preferably written rather than simply discussed. Reviewing with young persons their sense of self during this final phase of treatment is also important. Encouraging them to broaden this to include newly identified personal factors, in addition to appearance, such as "I am funny, creative, smart," can help to reinforce a healthier way of viewing themselves.

Parental involvement to support a young person's ongoing recovery from BDD is vital. Parents should be open and curious with their child about rituals or repetitive behaviors that may emerge in the future and monitor any new accommodating behaviors that they, or other family members, engage in. A relapse prevention plan should also include booster appointments with the young person's therapist, to provide ongoing support for them and their family. Living a life without BDD should be a point of discussion during the final stages of therapy, and the adolescent should be encouraged to expect that this is indeed within their future. 


\section{CONCLUSION}

BDD among adolescents is distressing and debilitating yet, to date, has drawn little research attention. Clinical understanding of the disorder, and risk factors associated with disorder onset, is in its infancy. Better clinical understanding is required to further develop treatment programs that may help young people most effectively. It is likely that the demand for treatment will continue to go unrecognized for some time, and significant effort needs to be directed toward raising awareness of this condition. There is a clear need to further develop and disseminate effective tools and methods for screening for this disorder in psychiatric and community settings, recognizing that many young people will not spontaneously disclose their symptoms, potentially because they do not feel their appearance concerns are inaccurate or delusional. Continuing to educate professionals on emerging treatments and new treatment strategies is also critical, as treatment development remains an important area of ongoing work.

Based on adult literature and clinical experience, we have identified the currently available treatment strategies for working with young people. We have highlighted key areas of treatment where adaptations can increase treatment success with young people. A thorough but simple psychoeducation phase, where BDD is distinguished from normal appearance concerns, and a shared formulation developed with families is important. Maintaining parental involvement throughout treatment also appears to be useful in supporting ongoing implementation of E/RP. Although cognitive strategies have comparatively limited use among younger people with BDD, visual perception training, such as attention training and mirror retraining, appear to be vital to recovery.

\section{REFERENCES}

Aldea, M. A., Storch, E. A., Geffken, G. R., \& Murphy, T. K. (2009). Intensive cognitive-behavioral therapy for adolescents with body dysmorphic disorder. Clinical Case Studies, 8(2), 113-121.

Buhlmann, U., Etcoff, N., \& Wilhelm, S. (2006). Emotion recognition bias for contempt and anger in body dysmorphic disorder. Journal of Psychiatric Research, 40(2), 105-111.

Buhlmann, U., McNally, R. J., Wilhelm, S., \& Florin, I. (2002). Selective processing of emotional information in body dysmorphic disorder. Journal of Anxiety Disorders, 16(3), 289-298.

Cash, T. F., \& Pruzinsky, T. (2002). Body image: A handbook of theory, research, and clinical practice. New York, NY: Guilford Press.

Enander, J., Andersson, E., Mataix-Cols, D., Lichtenstein, L., Alström, K., Andersson, G., Ljótsson, B. \& Rück, C. (2016). Therapist guided internet based cognitive behavioural therapy for body dysmorphic disorder: Single blind randomised controlled trial. British Medical Journal, 352, i241.

Geremia, G., \& Neziroglu, F. (2001). Cognitive therapy in the treatment of body dysmorphic disorder. Clinical Psychology and Psychotherapy, 8(4), 243-251.

Greenberg, J. L., Markowitz, S., Petronko, M. R., Taylor, C. E., Wilhelm, S., \& Wilson, G. T. (2010). Cognitivebehavioral therapy for adolescent body dysmorphic disorder. Cognitive and Behavioral Practice, 17(3), 248-258.

Greenberg, J. L., Mothi, S. S., \& Wilhelm, S. (2016). Cognitive-behavioral therapy for adolescent body dysmorphic disorder: A pilot study. Behavior Therapy, 47(2), 213-224.

Gunstad, J., \& Phillips, K. A. (2003). Axis I comorbidity in body dysmorphic disorder. Comprehensive Psychiatry, 44, 270-276.

Harrison, A., de la Cruz, L. F., Enander, J., Radua, J., \& Mataix-Cols, D. (2016). Cognitive-behavioral therapy for body dysmorphic disorder: A systematic review and meta-analysis of randomized controlled trials. Clinical Psychology Review, 48, 43-51. 
Krebs, G., de la Cruz, L. F., Monzani, B., Bowyer, L., Anson, M., Cadman, J., . . Mataix-Cols, D. (2017). Longterm outcomes of cognitive-behavioral therapy for adolescent body dysmorphic disorder. Behavior Therapy, 48(4), 462-473.

Krebs, G., Turner, C., Heyman, I., \& Mataix-Cols, D. (2012). Cognitive behaviour therapy for adolescents with body dysmorphic disorder: A case series. Behavioural and Cognitive Psychotherapy, 40(4), 452-461.

Lambrou, C., Veale, D., \& Wilson, G. (2011). The role of aesthetic sensitivity in body dysmorphic disorder. Journal of Abnormal Psychology, 120(2), 443-453.

Lambrou, C., Veale, D., \& Wilson, G. (2012). Appearance concerns comparisons among persons with body dysmorphic disorder and nonclinical controls with and without aesthetic training. Body Image, 9(1), 86-92. http://dx.doi.org/10.1016/j.bodyim.2011.08.001

Lebowitz, E. R., Panza, K. E., Su, J., \& Bloch, M. H. (2012). Family accommodation in obsessive-compulsive disorder. Expert Review of Neurotherapeutics, 12(2), 229-238.

Levine, M. P., \& Smolak, L. (2002). Body image development in adolescence. Body Image: A Handbook of Theory, Research, and Clinical Practice, 74-82.

Littleton, H. L., \& Ollendick, T. (2003). Negative body image and disordered eating behavior in children and adolescents: What places youth at risk and how can these problems be prevented? Clinical Child and Family Psychology Review, 6, 51-66.

Mataix-Cols, D., Fernández de la Cruz, L., Isomura, K., Anson, M., Turner, C., Monzani, B., ... Krebs, G. (2015). A pilot randomized controlled trial of cognitive-behavioral therapy for adolescents with body dysmorphic disorder. Journal of the American Academy of Child and Adolescent Psychiatry, 54(11), 895-904.

Mitchell, S. H., Petrie, T. A., Greenleaf, C. A., \& Martin, S. B. (2012). Moderators of the internalization-body dissatisfaction relationship in middle school girls. Body Image, 9, 431-440.

Nakatani, E., Mataix-Cols, D., Micali, N., Turner, C., \& Heyman, I. (2009). Outcomes of cognitive behaviour therapy for obsessive compulsive disorder in a clinical setting: A 10-year experience from a specialist OCD service for children and adolescents. Child and Adolescent Mental Health, 14(3), 133-139.

Neziroglu, F., Khemlani-Patel, S., \& Veale, D. (2008). Social learning theory and cognitive behavioral models of body dysmorphic disorder. Body Image, 5, 28-38.

Neziroglu, F., McKay, D., Todaro, J., Yaryura-Tobias, J. A. (1996). Effect of cognitive behavior therapy on persons with body dysmorphic disorder and comorbid Axis II diagnosis. Behavior Therapy, 27, 67-77.

Neziroglu, F., Roberts, M., \& Yaryuya-Tobias, J. A. (2004). Behavioural model for body dysmorphic disorder. Psychiatric Annals, 34(12), 915-920.

Osman, S., Cooper, M., Hackmann, A., \& Veale, D. (2004). Spontaneously occurring images and early memories in people with body dysmorphic disorder. Memory, 12, 428-436.

Phillipou, A., Rossell, S. L., Wilding, H. E., \& Castle, D. J. (2016). Randomised controlled trials of psychological \& pharmacological treatments for body dysmorphic disorder: A systematic review. Psychiatry Research, 245, 179-185.

Phillips, K. A., \& Diaz, S. F. (1997). Gender differences in body dysmorphic disorder. Journal of Nervous and Mental Disease, 185(9), 570-577.

Phillips, K. A., Didie, E. R., Menard, W., Pagano, M. E., Fay, C., \& Weisberg, R. B. (2006). Clinical features of body dysmorphic disorder in adolescents and adults. Psychiatry Research, 141, 305-314.

Phillips, K. A., Pagano, M. E., Menard, W., \& Stout, R. L. (2006). A 12-month follow-up study of the course of body dysmorphic disorder. The American Journal of Psychiatry, 163(5), 907-912.

Rabiei, M., Mulkens, S., Kalantari, M., Molavi, H., \& Bahrami, F. (2012). Metacognitive therapy for body dysmorphic disorder patients in Iran: Acceptability and proof of concept. Journal of Behavior Therapy and Experimental Psychiatry, 43(2), 724-729.

Rief, W., Buhlmann, U., Wilhelm, S., Borkenhagen, A., \& Brähler, E. (2006). The prevalence of body dysmorphic disorder: A population-based survey. Psychological Medicine, 36(06), 877-885.

Rodin, J., Silberstein, L., \& Striegel-Moore, R. (1984). Women and weight: A normative discontent. Nebraska Symposium on Motivation, 32, 267-307. 
Rosen, J. C., Reiter, J., \& Orosan, P. (1995). Cognitive-behavioral body image therapy for body dysmorphic disorder. Journal of Consulting and Clinical Psychology, 63(2), 263.

Schneider, S. C., Mond, J., Turner, C. M., \& Hudson, J. L. (2017a). Sex differences in the presentation of body dysmorphic disorder in a community sample of adolescents. Journal of Clinical Child and Adolescent Psychology. Advance online publication. http://dx.doi.org/10.1080/15374416.2017.1321001

Schneider, S. C., Mond, J., Turner, C. M., \& Hudson, J. L. (2017b). Subthreshold body dysmorphic disorder in adolescents: Prevalence and impact. Psychiatry Research, 251, 125-130.

Schneider, S. C., Turner, C. M., Mond, J., \& Hudson, J. L. (2017). Prevalence and correlates of body dysmorphic disorder in a community sample of adolescents. Australian \& New Zealand Journal of Psychiatry, 51(6), 595-603.

Turner, C. M., Mataix-Cols, D., Lovell, K., Krebs, G., Lang, K., Byford, S., \& Heyman, I. (2014). Telephone cognitive-behavioral therapy for adolescents with obsessive-compulsive disorder: a randomized controlled non-inferiority trial. Journal of the American Academy of Child \& Adolescent Psychiatry, 53(12), 1298-1307.

Veale, D. (2002a). Over-valued ideas: A conceptual analysis. Behaviour Research and Therapy, 40(4), 383-400.

Veale, D. (2002b). Shame in body dysmorphic disorder. In P. Gilbert \& J. Miles (Eds.), Body shame: Conceptualisation, research and treatment (pp. 267-283). Hove, United Kingdom: Brunner-Routledge.

Veale, D. (2004). Advances in a cognitive behavioural model of body dysmorphic disorder. Body Image, 1 , $113-125$.

Veale, D., Anson, M., Miles, S., Pieta, M., Costa, A., \& Ellison, N. (2014). Efficacy of cognitive behaviour therapy versus anxiety management for body dysmorphic disorder: A randomised controlled trial. Psychotherapy and Psychosomatics, 83, 341-353.

Veale, D., Gournay, K., Dryden, W., Boocock, A., Shah, F., Willson, R., \& Walburn, J. (1996). Body dysmorphic disorder: A cognitive behavioural model and pilot randomised controlled trial. Behaviour Research and Therapy, 34(9), 717-729. http://dx.doi.org/10.1016/0005-7967(96)00025-3

Veale, D., \& Neziroglu, F. (2010). Body dysmorphic disorder: A treatment manual. Hoboken, NJ: Wiley.

Wilhelm, S., Buhlmann, U., Hayward, L. C., Greenberg, J. L., \& Dimaite, R. A. (2010). A cognitive-behavioral treatment approach for body dysmorphic disorder. Cognitive and Behavioral Practice, 17, 241-247.

Wilhelm, S., Phillips, K. A., Fama, J. M., Greenberg, J. L., \& Steketee, G. (2011). Modular cognitive-behavioral therapy for body dysmorphic disorder. Behavior Therapy, 42, 624-633.

Wilhelm, S., Phillips, K. A., \& Steketee, G. (2013). A cognitive-behavioral treatment manual for body dysmorphic disorder. New York, NY: Guilford Press.

Wilhelm, S., Phillips, K. A., Didie, E., Buhlmann, U., Greenberg, J. L., Fama, J. M., Keshaviah, A., \& Steketee, G. (2014). Modular cognitive-behavioral therapy for body dysmorphic disorder: A randomized controlled trial. Behavior Therapy, 45(3), 314-327.

Willson, R., Veale, D., \& Freeston, M. (2016). Imagery rescripting for body dysmorphic disorder: A multiplebaseline single-case experimental design. Behavior Therapy, 47(2), 248-261.

Correspondence regarding this article should be directed to Cynthia Turner, PhD, Australian Catholic University, School of Psychology, 1100 Nudgee Rd., Banyo, Brisbane, Australia.E-mail: Cynthia.turner@acu.edu.au 